\title{
FUZZY MODELS OF WEB SERVICES SELECTION IN THE DEVELOPMENT OF SERVICE-ORIENTED INFORMATION SYSTEMS FOR SMALL AND MEDIUM ENTERPRISES
}

\author{
Irina Shpolianskaya ${ }^{1, a, *}$, Alexei Dolzhenko ${ }^{2, b}$ and Sergey Glushenko ${ }^{3, c}$ \\ ${ }^{1}$ Rostov State University of Economics, Department of Information Systems and Applied Informatics, \\ Bolshaya Sadovaya str., 69, 344002 Rostov-on-Don, Russia \\ ${ }^{2}$ Rostov State University of Economics, Department of Information Systems and Applied Informatics, \\ Bolshaya Sadovaya str., 69, 344002 Rostov-on-Don, Russia \\ ${ }^{3}$ Rostov State University of Economics, Department of Information Systems and Applied Informatics, \\ Bolshaya Sadovaya str., 69, 344002 Rostov-on-Don, Russia \\ airinaspol@yandex.ru, bdoljenkoalex@gmail.com, ${ }^{c}$ gs-gears@yandex.ru \\ *Corresponding author
}

Cite as: Shpolianska, I., Dolzhenko, A., Glushenko, S. (2017). Fuzzy models of web services selection in the development of service-oriented information systems for small and medium enterprises, Ekonomicko-manazerske spectrum, 11(2), 64-75.

Available at: dx.doi.org/10.26552/ems.2017.2.64-75

\begin{abstract}
Nowadays, small and middle enterprises (SME's) competitiveness increasingly depends on their ability to adapt successfully to a rapidly changing business environment. Because of this information system that provides integrated support for all business procceses of an enterprise must be highly dynamic and flexible. Service-oriented architecture (SOA) considered as a set of interacting web services is able to ensure the information system flexibility, reduce software development, maintenance and upgrades costs, and thereby increase responsiveness of the system to changing business requirements. In service-oriented design web-services have a direct impact on the possibility of development, adaptation and modernization of the information system. The paper discusses principles and methods for SOA design for SME's considering the structure of business processes as a basis of coordinated service interactions. Taking into account the business requirements the problem of web services optimal selection for service-oriented information system is solved. The problem of web service selection is formulated as a problem of choice with fuzzy preferences. Fuzzy logic and expert methods are used for evaluation and selection of web services. The developed procedures allow the user to evaluate and select from a set of available services the services with the required functionality and quality. The proposed fuzzy model and algorithms of web services selection provide the effective decision-support mechanisms for construction of SOA-based systems for small and middle enterprises. The new approach helps SME's to strengthen competitiveness through developing the information systems with flexible structure that allows to consider all changes in business environment, and reduce the time-tomarket with the system.
\end{abstract}

Keywords: small and medium-sized enterprises (SMEs), management information system, service-oriented architecture, web services selection, fuzzy models

JEL Classification: M15, L86, F61 


\section{Introduction}

The need for integration of business processes and their information flows is growing in modern business. Small and medium-sized enterprises are forced to react quickly to a changing environment and adapt their business processes. In order to achieve the required flexibility enterprises must integrate heterogeneous applications and systems in order to support the effective business process management. (Seta \& Arman, 2014) Service-oriented architecture (SOA) is a growing trend in the information systems development. It provides business with components and services as functional units of information system which can be loosely coupled and which can communicate using standardized protocols. (Stal, 2002) SOA approach offers great benefits to SMEs including business process flexibility, improved time to market, reduced total cost of ownership, and business risk. (Ray \& Ray, 2006) In recent times web services are increasingly used for composite applications in service oriented architectures. Web service technology is identified as a key driver for SMEs to gain operational and strategic benefit from net-sourced information systems. (Vidgen et al., 2004) This software architecture is represented as a set of interrelated services and building blocks that collectively support an organization's goals and business processes. (Papazoglou \& Georgakopoulos, 2003) Properties of the composite application directly depend on the quality characteristics of web services that are integrated into the system. Thus, the selection of appropriate and reliable services is of great importance. The services claimed by the provider, can be used by one or more clients. On the other hand, each customer can choose one particular service from the many available services.

Increasing number of Web services on the Internet requires effective criteria and selection methods that will allow users to make the best choice. (Zheng et al., 2011) A typical procedure for selecting appropriate services from a set of alternatives for subsequent integration into the information system is based on requirements that describe the quality of service (QoS). (Zeng et al., 2004) When modelling an information system with SOA architecture user performs a selection of appropriate service from a set of available comparable services with QoS non-functional requirements such as performance, reliability, security, response time etc. (Menasce, 2002; Ouzzani \& Bouguettaya, 2004; Rosario et al., 2008) Many non-functional properties of services such as response time, are stochastic in nature. Dynamic environment of service deployment due to varying network conditions, latencies and server overload can lead to deviation of values of non-functional service quality indicators. (Hwang et al., 2007) Some services in the particular envocation may require significantly more execution time, which in turn will increase the Total Cost of Ownership.

A number of methods based on QoS characteristics for appropriate services selection from a set of candidates with similar functions were developed. Most of these methods dedicated to the analysis of non-functional characteristics of web services as the main factors influencing the service selection decision. In Zheng et al. (2013) authors offered a systematic approach for calculating the QoS for composite services with complex structures, taking into account the probability and conditions of each execution. Maximilien and Singh (2004) presented a dynamic service selection approach via an agent framework coupled with the QoS ontology. With this approach, participants can collaborate to determine the quality and reliability of service to each other. In Kritikos and Plexousakis (2009) a selection method based on ranking approach was presented. The proposed algorithm filters avalable services according to the user preferences and chooses which service can be most probably used through the predefined categorizer. Skoutas et al. (2010) proposed a methodology for ranking the relevant services for a given request, introducing objective measures for clustering the relevant services based on dominance relationships defined among the services. The proposed methods perform the 
matching processes by employing multiple criteria and two types of clustering algorithms: Approximate Skyline Clustering and Heuristic Skyline Clustering. The work in $\mathrm{Yu}$ and Bouguettaya (2010) presents a novel concept, called p-dominant service skyline. A provider S belongs to the $\mathrm{p}$-dominant skyline if the chance that $\mathrm{S}$ is dominated by any other provider is less than p. In Yu and Bouguettaya (2011) authors propose a multi-attribute optimization approach and a set of service skyline computation techniques that return a set of most suitable service providers.

The work in Ardagna and Pernici (2007) presented an optimization approach for web services composition using dynamic service selection through adaptive reoptimization under variable QoS characteristics of the web services. Here the problem of web services selection is formalized as a problem of integer linear programming. In Ardagna and Mirandola (2010) service selection problem is formulated as a constrained non-linear multi-criteria optimization problem and a heuristic algorithm is proposed to determine a scalable and efficient solution. The framework in Ardagna et al. (2011) exploits a new optimization approach based on mixed integer linear programming models, which supports the selection of stateful Web services under severe QoS constraints. If a feasible solution of the optimization problem does not exist, the approach negotiates the QoS parameters with the service providers to find a suboptimal solution. Yu et al. (2007) has proposed service selection methods with maximum utility function with QoS constraints. The methods used are Multidimensional multi choice 01 knapsack problem and graph based model. To solve this problem that is clearly NP-hard Menasce et al. (2010) presents an optimized heuristic algorithm that finds the optimal solution without having to explore the entire solution space.

Recently, evolutionary algorithms were proposed to tackle the web service selection problem. So Ma et al. (2008) have proposed a Genetic Algorithm approach to solve the scalability problem for the service selection problem. The work in Klein et al. (2014) presented self-adaptive genetic algorithm, which is used to determine the quality of web service, and in Zhang et al. (2010) for these purposes has been proposed the ant colony algorithm. However, the functional aspects also have a significant impact. In addition, performance characteristics, which are determined directly during exploitation of information system may also have an impact on the effectiveness of service-oriented architecture. In Zheng et al. (2011) a collaborative filtering approach have been proposed for predicting QoS values of web services and making web service recommendation by taking advantages of past usage experiences of service users. In Hao et al. (2010) an information retrieval approach was suggested for discovering and ranking web services automatically, given a textual description of the desired services. The aim of this work is to develop a modeling method for evaluating the web service quality for subsequent service selection and composition in SOA. The problem of web service selection is formulated as a problem of choice with fuzzy preferences. This approach can serve as a basis for the further development of decision-making software that supports automatic modeling of SOA systems.

\section{Methodology}

The web service selection methods often use a measure of quality of service (QoS). (Menasce (2002)) This measure includes such characteristics as reliability, security, trust and execution cost, and is mainly related to non-functional characteristics of services. However, consumers are equally interested in the best possible performance of services, based on both functional and non-functional characteristics for the successful deployment and operation of service-oriented architecture. Evaluation of functional characteristics when selecting web 
services is more complex procedure than procedures for evaluation of non-functional requirements. Functional requirements in many cases are presented in a simplified form, and there is a need for a subjective user satisfaction assessment of service quality and compliance assessment with the requirements. In the management of service-oriented system functional characteristics of web-services have a direct impact on the possibility of development, adaptation and modernization of the information system. In general, it is quite difficult to find quantitative indicators of the functional requirements. So an approach to web service conformity assessment based on the domain ontology with required structure of business processes has been developed. (Maximilien \& Singh, 2004)

Evaluation of the effectiveness of the system based on the selected structure can be obtained only after the application is deployed and will be away for some time. These circumstances raise the need for formulation of the web services selection problem as decision making under uncertainty enabled by fuzzy logic techniques. Wang et al. (2007) proposes a fuzzy-based UDDI with QoS support. The proposed method considers not only the objective factors described by service providers but also the subjective information with trustability evaluations from users who use those services. Genetic algorithm is adapted to learn user preferences, and fuzzy logic is applied for making decisions. Wang in (2009) has proposed a resolution process for determining the linguistic weights of QoS criteria based on a group of participants' preferences. The dynamic composition of QoS based web service selection is done using Fuzzy constraint satisfaction problem. The work in Wang et al. (2010) presented a QoS-aware service selection model based on fuzzy linear programming technologies, in order to assist service consumers in selecting most suitable services with consideration of their expectations and preferences.

\subsection{A fuzzy linguistic method for web service quality assessment}

We propose a novel approach to the problem of web service quality evaluation which we dene in terms of fuzzy preference relations and linguistic assessment variables. The quality of service is formalized as follows:

$\mathrm{FMG}=\langle\mathrm{G}, \mathrm{L}, \mathrm{P}, \mathrm{A}\rangle$,

where $\quad \mathrm{G}$ is a graph, where each vertex $F_{j}\left(j=0, \ldots, N_{D}\right)$ is associated with linguistic variable $x_{j}^{i} \in L_{j}$, that describes a specific service quality indicator; $L=\left\{L_{j}\left(j=0, \ldots, N_{D}\right)\right\}$ is a set of linguistic values that $x_{j}^{i} \in L_{j}$ takes; $\mathrm{P}$ is a fuzzy preference relation; $A$ is an aggregation operator.

When using ternary fuzzy classifier on the scale $[0,1]$ values $L_{j}$ should be as follows:

\{ Low level (L), Middle level (M), High level $(H)\}$.

Fuzzy preference relations are defined as follows:

$$
P=\left\{F_{i}(\varphi) F_{j} \mid \varphi \in(\succ, \approx)\right\}
$$

where $\succ$ denotes strict preferences, $\approx$ denotes indifference.

To obtain an integrated linguistic assessment of the web service quality we use aggregation operator for every non-terminal vertex of the graph. The aggregator uses the assessment of the quality indicators represented as child nodes in the tree. The ordered weighted averaging operator (Yager's OWA operator) (Yager (1988)) and Fishburn coefficients (Adelson and Fishburn (1971)), as associated collection of weights, are used for aggregation. The Fishburn coefficients are defined as follows: $p_{i}=r_{i} / \sum_{j=1}^{N} r_{j}$, where $i=\overline{1, N}$ and $N$ is a number of child 
vertices of the graph involved in information aggregation; $r_{i}$ is defined on the basis of preference relations.

If for each indicator $\left(F_{k .1} \ldots F_{k . N}\right)$ on the selected graph level $k$ linguistic values $L=\left(L_{k .1} \ldots\right.$ $\left.L_{k . N}\right)$ and weighting coefficients $p_{k}=\left(p_{k .1}, \ldots, p_{k . N}\right)$ are determined, then aggregation operator represents the linguistic assessment of the membership function, determined for 01-classifier:

$$
\mu_{k}(x)=\sum_{i=1}^{N} \mu_{k, i}(x) p_{i}, i=\overline{1,3}
$$

The evaluation of trapezoidal membership function $\mu_{k}(x)$ can be done as operation with vertices of graph as functions $\mu_{k . i}(x)$. Function $\mu_{k}(x)$ is used to assess linguistic value of $F_{k}$ indicator. For ternary fuzzy 01-classifier it is necessary to define minimum distance between $\mu_{k}(x)$ and $\mu_{i}(x), i=\overline{1,3}$ on the basis of minimum distance $\rho_{k i}$ between a fuzzy set defined by the membership function $\mu_{k}(x)$, and each of fuzzy sets corresponding to membership functions $\mu_{i}(x), i=\overline{1,3}$.

To estimate distance between fuzzy set A and set B absolute or relative Hamming distance as well as squared Euclidean distance can be used. For indicator $F_{k}$ that is defined by trapezoidal membership function with parameters $a_{L}^{k}, a_{1}^{k}, a_{2}^{k}, a_{R}^{k}$ and membership functions $\mu_{i}(x), i=\overline{1,3}$ that are also trapezoidal with parameters $\left(b_{L}^{i}, b_{1}^{i}, b_{2}^{i}, b_{R}^{i},\right)$, the distance between fuzzy sets is calculated as follows:

$$
\rho_{k i}=\max \left\{\left|a_{L}^{k}-b_{L}^{i}\right|,\left|a_{1}^{k}-b_{1}^{i}\right|,\left|a_{2}^{k}-b_{2}^{i}\right|,\left|a_{R}^{k}-b_{R}^{i}\right|\right\}
$$

The matching value $F_{k}$ of a specific linguistic level of the ternary scale on 01-classifier is defined as $\min \left(p_{k i}\right)$. The procedure of factors aggregation is to be performed for each not-end vertices down up to obtain linguistic value of web service quality. The formation of the integral quality index F0 for graph G is the aggregation of factors for each of the non-terminal graph vertices from the leaves to the top.

The algorithm for integrated quality assessment of web services is as follows.

1. Form a graph $\mathrm{G}$ of the web service quality indicators with vertices $F j\left(j=0, \ldots, N_{D}\right)$.

2. Form a system of preference relations $P=\left\{F_{i}(\varphi) F_{j} \mid \varphi \in(\succ, \approx)\right\}$ between graph vertices on the basis of expert assessments.

3. For each of the end vertices in the graph form a set of linguistic values of factor levels.

4. For non-end vertices in the graph apply the aggregation operator to compute their linguistic values.

As a result, we obtain a linguistic evaluation $F$ for the root vertex of the graph, which characterizes the integral quality of the web service, and for intermediate non-end vertices the quality of service concerning certain group of indicators, for example its functionality.

\subsection{A fuzzy model of web service selection in service-oriented architecture}

Now that we have obtained integrated web service quality assessment we are able to solve the problem of service selection and composition in SOA.

Consider a fuzzy-set based model of web service selection for service-oriented information system integration.

Let us consider the functional structure of a management information system that includes a set of business processes $X=\left\{x_{1}, x_{2}, \ldots, x_{i}, \ldots, x_{n}\right\}, i=\overline{1, n}$. Let a set of web services 
$Y=\left\{y_{1}, y_{2}, \ldots, y_{j}, \ldots, y_{m}\right\}, \quad j=\overline{1, m}$, that implement the functionality of enterprise business processes is available to information system developer. The possibility of using a web service $y_{j}$ with a given quality level for business process $x_{i}$ will be provided as fuzzy set $\mathrm{B}$, which is defined with its membership function $\mu_{B}(x, y) \in[0,1]$. This function shows how the quality of a web service $y_{j}$ meets the requirements of the business process $x_{i}$. Fuzzy relation matrix that represents fuzzy set $B$ is as follows:

$$
B=\left\|\begin{array}{cccc}
\mu_{B}\left(x_{1}, y_{1}\right) & \mu_{B}\left(x_{1}, y_{2}\right) & \ldots & \mu_{B}\left(x_{1}, y_{m}\right) \\
\mu_{B}\left(x_{2}, y_{1}\right) & \mu_{B}\left(x_{2}, y_{2}\right) & \ldots & \mu_{B}\left(x_{2}, y_{m}\right) \\
\ldots & \ldots & \ldots & \ldots \\
\mu_{B}\left(x_{n}, y_{1}\right) & \mu_{B}\left(x_{n}, y_{2}\right) & \ldots & \mu_{B}\left(x_{n}, y_{m}\right)
\end{array}\right\|
$$

Each business process $x_{i}, \quad i=\overline{1, n}$ of the information system can be supported with one or multiple web services from $\left\{y_{j}\right\} \quad j=\overline{1, m}$, but one and the same web service cannot support more than one business process. This means that in each column of the matrix only one value of the membership function $\mu_{B}\left(x_{i}, y_{j}\right)$ can be greater than 0 .

When you assign web service $y_{j}$ to business process $x_{i}$ you must take into account QoS constraints. These constraints may be determined by the permissible levels of service quality. To define such restriction we will form a $\Delta$-level subset of a fuzzy set $B$.

Let us define the $\Delta$-level as a vector:

$$
\Delta=\left\{\delta_{1}, \delta_{2}, \ldots, \delta_{i}, \ldots, \delta_{n}\right\}=\left\{\delta_{i}\right\}, \delta_{i} \in[0,1], i=\overline{1, n}
$$

$\Delta$-level subset $B_{\Delta}$ of a fuzzy set $B$ we define as follows:

$$
B_{\Delta}=\left\{x_{i} \in X, y_{j} \in Y,: \forall j\left(\mu\left(x_{i}, y_{j}\right) \geq \delta_{i}, i=\overline{1, n}, j=\overline{1, m}\right)\right\}
$$

Levels $\delta_{i}, \quad i=\overline{1, n}$ specify the minimum requirements for service quality. Then we define a criterion of effective selection of web service $y_{j}, \quad j=\overline{1, m}$ for business process $x_{i}, \quad i=\overline{1, n}$ as a projection $B_{X}^{\max }$ of binary fuzzy relation $B_{\Delta}$, which has the following membership function

$$
\mu_{B_{X}^{\max }}(x)=\sup _{Y} \mu_{B_{\Delta}}(x, y)
$$

In the case of selecting web services with minimum sufficient quality for information system, we will use a projection $B_{X}^{\min }$ with membership function:

$$
\mu_{B_{X}^{\min }}(x)=\inf _{Y} \mu_{B^{M}}(x, y)
$$

In order to eliminate the influence of zero values of the membership function $\mu_{B_{\Delta}}\left(x_{i}, y_{j}\right)$ we will form a modified matrix $B^{M}$, based on $B_{\Delta}$ :

$$
\mu_{\mathrm{B}}^{M}\left(x_{i}, y_{j}\right)=\left\{\begin{array}{cc}
\mu_{B_{\Delta}}\left(x_{i}, y_{j}\right), & \mu_{B_{\Delta}}\left(x_{i}, y_{j}\right)>0 \\
1,1, & \mu_{B_{\Delta}}\left(x_{i}, y_{j}\right)=0 .
\end{array}\right.
$$

Thus, the problem of service-oriented modeling is presented as modified assignment problem that helps to select the most effective available web service for each business process of the enterprise. The proposed algorithm for fuzzy model of web services selection problem will be presented as follows.

1. Define the set of business processes $X$ according to the structure of the enterprise information system.

2. Define the set of available web services $Y$. 
3. Evaluate with the help of independent external experts how effectively business process $x_{i}$ can be implemented with web service $y_{j}$. Expert assessments are presented in the form of fuzzy relation matrix $B$ by (9).

4. Form $\Delta$-level vector according to user requirements and acceptable levels of web service quality for each business process.

5. Form matrix $B_{\Delta}$ as a $\Delta$-level subset of a fuzzy set $B$ by (7).

6. Define the projection $B_{X}^{\max }$ by (8) or $B_{X}^{\min }$ by (9) and fuzzy set matrix $B_{\Delta}^{\text {eff }}$ for the most effective selection of web service $y_{j}$ for business process $x_{i}:\left(x_{i} \rightarrow y_{j}\right)$.

Membership function of fuzzy relation matrix $B_{\Delta}^{e f f}$ is defined as follows:

$$
\mu_{\Delta}^{e f f}\left(x_{i}, y_{j}\right)=\left\{\begin{array}{cc}
\mu_{\Delta}\left(x_{i}, y_{j}\right), & \mu_{\Delta}\left(x_{i}, y_{j}\right)=\mu_{B_{X}}\left(x_{i}\right), \\
0, & \mu_{\Delta}\left(x_{i}, y_{j}\right) \neq \mu_{B_{X}}\left(x_{i}\right) .
\end{array}\right.
$$

Nonzero elements of the matrix $B_{\Delta}^{\text {eff }}$ define the assignment of web service $y_{j}$ for business process $x_{i},\left(x_{i} \rightarrow y_{j}\right)$. The degree of confidence that web service $y_{j}$ will ensure a given level of service quality for business process $x$ is characterized by the value of the membership function $\mu_{\Delta}\left(x_{i}, y_{j}\right)$.

\section{Results}

Let's look at an example of using the fuzzy models of web service selection on the base of integrated web service quality indicator.

\subsection{An example of using the fuzzy model of web service quality assessment}

Here we consider the following indicator system to measure a web service quality. $F_{0}\left\{F_{11}\right.$ $\left.F_{1.1}, F_{1.2}, F_{1.3}\right\}, F_{2}\left\{F_{2.1}\left\{F_{2.1 .1}, F_{2.1 .2}, F_{2.1 .3}, F_{2.1 .4}\right\}, F_{2.2}\left\{F_{2.2 .1}, F_{2.2 .2}, F_{2.3 .3}\right\}\right\}$, where $\mathrm{F} 0$ is the integral web service quality indicator; F1 - indicator of the functional characteristics; F1.1 accuracy, as a measure of matching similarities between the service response and required result; F1.2 - robustness, the sustainability of the service to incorrect input data, or incorrect invocation sequence; F1.3 - interoperability, the ability of the service to provide seamless and automatic connections to another software application; F1.4 -availability, the ability of a service to perform a required function within a specified period of time; F2 - indicator of nonfunctional characteristics; F2.1 - indicators of operating performance; F2.1.1 - performance of the service; F2.1.2 - reliability of a web service, the probability of successful execution of the business process using the service; F2.1.3 - scalability, the ability of the service to increase its productivity if necessary; F2.1.4 - security, that includes user authentication, message integrity and confidentiality; F2.2 - web service user satisfaction indicators in terms of business; F2.2.1 - cost of invocation and using of web service; F2.2.2 - reputation of the service as a measure of the reliability of the supplier; F2.2.3 - an actual measure of user's experience with provider in terms of delivered quality.

For a given system experts were asked to provide preference rankings of web service quality indicators. As a result we have obtained the following system of preferences:

$$
\begin{aligned}
& P=\left\{F_{1} \succ F_{2} ; F_{1.1} \succ F_{1.2} \approx F_{1.3} \approx F_{1.4} ;\right. \\
& \left.F_{2.1} \succ F_{2.2} ; F_{2.1 .1} \approx F_{2.1 .2} \approx F_{2.1 .4} \succ F_{2.1 .3} ; F_{2.2 .1} \succ F_{2.2 .2} \approx F_{2.2 .3}\right\} \text {. }
\end{aligned}
$$

For the selected factors experts have formed linguistic values of factor levels that define quality of web service:

$$
\begin{gathered}
F_{1.1}-H ; F_{1.2}-H ; F_{1.3}-M ; F_{1.4}-M ; F_{2.1 .1}-H ; F_{2.1 .2}-M ; \mathrm{F}_{2.1 .3}-\mathrm{L} ; \mathrm{F}_{2.1 .4}-\mathrm{L} ; \mathrm{F}_{2.2 .1}-\mathrm{H} ; \\
F_{2.2 .2}-L ; F_{2.2 .3}-L .
\end{gathered}
$$


The results of fuzzy modeling are shown in table 1. Linguistic variables with levels High (H), Medium (M) and Low (L) are given by trapezoidal membership function.

Table 1: The results of modelling

\begin{tabular}{llllll}
\hline Name of indicator & The level of indicator & \multicolumn{3}{l}{ Trapezoidal number } & \\
\hline$F_{0}$ & $\mathrm{H}$ & 0.70 & 0.80 & 0.85 & 1.00 \\
$F_{1}$ & $\mathrm{H}$ & 0.77 & 0.82 & 0.88 & 1.00 \\
$F_{2}$ & $\mathrm{M}$ & 0.25 & 0.45 & 0.55 & 0.75 \\
$F_{2.1}$ & $\mathrm{M}$ & 0.26 & 0.45 & 0.56 & 0.76 \\
$F_{2.2}$ & $\mathrm{M}$ & 0.25 & 0.45 & 0.55 & 0.75 \\
\hline
\end{tabular}

Source: Authors' calculations

It can be seen that the integral web service quality indicator and indicator of the functional characteristics can be assessed as "H - High", and the level of some non-functional indicators as "M - Medium". Thus, the analysis of web service quality shows an insufficient level of non-functional service characteristics.

\subsection{An example of using the fuzzy model for web service selection problem}

Let us see the process of SOA structure identification that will automate a set of business processes $X=\left\{x_{1}, x_{2}, x_{3}\right\}$. Let the information system developer has a set of available web services: $Y=\left\{y_{1}, y_{2}, y_{3}, y_{4}, y_{5}\right\}$.

A fuzzy set $B$ was formed on the basis of web services quality indicators and expert evaluation of how the quality of a web service $y_{j}$ meets the requirements of the business process $y_{j}$. Fuzzy set matrix $B$ is given below.

$$
B=\left\|\begin{array}{ccccc}
0 & 1 & 0 & 0 & 0 \\
0.6 & 0 & 0 & 0 & 1 \\
0 & 0 & 0.8 & 0.9 & 0
\end{array}\right\|
$$

Fuzzy graph that describes alternatives of web services selection for business processes according to fuzzy set matrix B is shown in Figure 1.

Figure 1: Fuzzy graph for web services selection

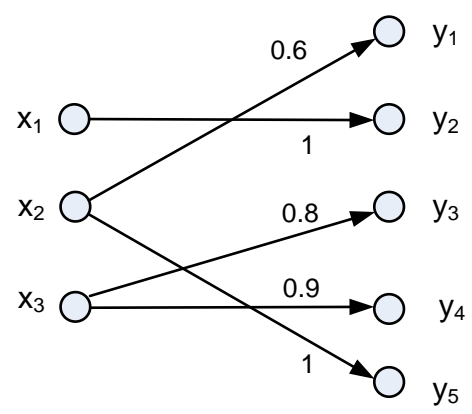

Source: Authors' calculations

There are four alternatives for construction of service-oriented information system:

1) $\mathrm{x} 1 \rightarrow \mathrm{y} 2, \mathrm{x} 2 \rightarrow \mathrm{y} 1, \mathrm{x} 3 \rightarrow \mathrm{y} 3$;

2) $\mathrm{x} 1 \rightarrow \mathrm{y} 2, \mathrm{x} 2 \rightarrow \mathrm{y} 5, \mathrm{x} 3 \rightarrow \mathrm{y} 3$;

3) $\mathrm{x} 1 \rightarrow \mathrm{y} 2, \mathrm{x} 2 \rightarrow \mathrm{y} 1, \mathrm{x} 3 \rightarrow \mathrm{y} 4$;

4) $\mathrm{x} 1 \rightarrow \mathrm{y} 2, \mathrm{x} 2 \rightarrow \mathrm{y} 5, \mathrm{x} 3 \rightarrow \mathrm{y} 4$. 
Now we will use a $\Delta$-level subset $\mathrm{B}_{\Delta}$ of a fuzzy set $\mathrm{B}$ to reduce the number of options under consideration while constructing the service-oriented architecture.

Let the $\Delta$-level vector $\Delta=\left\{\delta_{1}, \delta_{2}, \ldots, \delta_{i}, \ldots, \delta_{n}\right\}$ according to (2) has such values:

$$
\delta 1=0,9 ; \delta 2=0,8 ; \delta 1=0,8 \text {. }
$$

Then a $\Delta$-level subset $\mathrm{B}_{\Delta}$ according to (3) will be as follows:

$$
B_{\Delta}=\left\|\begin{array}{ccccc}
0 & 1 & 0 & 0 & 0 \\
0 & 0 & 0 & 0 & 1 \\
0 & 0 & 0.8 & 0.9 & 0
\end{array}\right\|
$$

Fuzzy graph of SOA model with constraints on QoS-levels is shown in Figure 2.

Figure 2: Fuzzy graph of SOA model with constraints on services levels

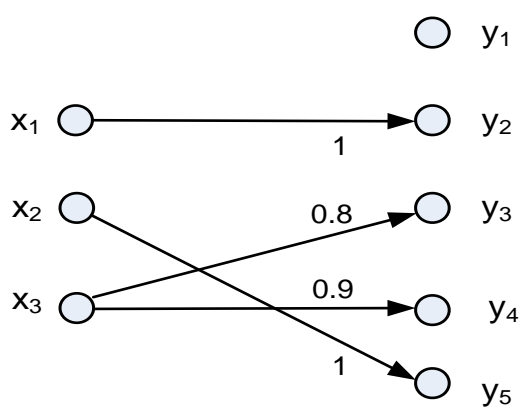

Source: Authors' calculations

As a result of construction of $\Delta$-level subset $\mathrm{B} \Delta$ of a fuzzy set $\mathrm{B}$ the number of options has been reduced by 2 cases:

1) $\mathrm{x} 1 \rightarrow \mathrm{y} 2, \mathrm{x} 2 \rightarrow \mathrm{y} 5, \mathrm{x} 3 \rightarrow \mathrm{y} 3$;

2) $\mathrm{x} 1 \rightarrow \mathrm{y} 2, \mathrm{x} 2 \rightarrow \mathrm{y} 5, \mathrm{x} 3 \rightarrow \mathrm{y} 4$.

For the final identification of the most effective web services for SOA system you would apply the selection criteria by (4). The result is a projection $B_{X}^{\max }=\left[\begin{array}{lll}1 & 1 & 0.9\end{array}\right]^{\mathrm{T}}$. The matrix of efficient web services selection for SOA is as follows:

$$
B_{\Delta}^{e f f}=\left\|\begin{array}{ccccc}
0 & 1 & 0 & 0 & 0 \\
0 & 0 & 0 & 0 & 1 \\
0 & 0 & 0 & 0.9 & 0
\end{array}\right\|
$$

Figure 3 shows the obtained fuzzy graph of the information system SOA structure with the highest level of web services quality: $\mathrm{x} 1 \rightarrow \mathrm{y} 2, \mathrm{x} 2 \rightarrow \mathrm{y} 5, \mathrm{x} 3 \rightarrow \mathrm{y} 4$.

Figure 3: Fuzzy graph of the SOA structure with the highest level of web services quality

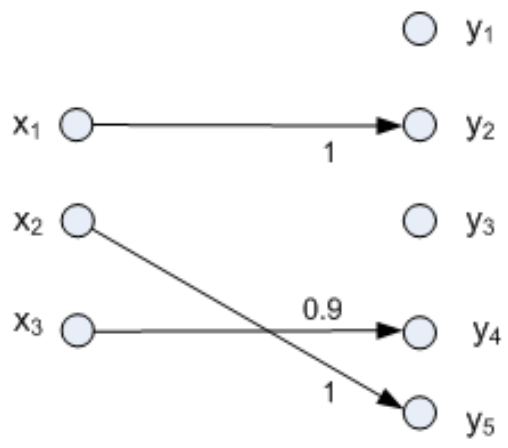

Source: Authors' calculations 
This example illustrates the possibility of using fuzzy modeling approach for designing a service-oriented architecture of information system with modified assignment problem algorithm, that allows developers to select the most effective web service for each business process of the enterprise system. (Dolzhenko, 2007)

\section{Discussion}

The problem of effective web service selection for SME's service-oriented information systems is presented as a decision-making problem under conditions of uncertainty that must take into account both functional (qualitative) and non-functional (quantitative and qualitative) characteristics of the services. We proposed a fuzzy logic approach for web service quality assessment with qualitative and quantitative indicators for their selection and composition in SOA system. Fuzzy logic models, on the one hand, help to integrate the effect of qualitative and quantitative indicators. On the other hand, they operate with linguistic variables ("low", "medium", "high") that help to assess user expectations about web services quality and how SOA system meets the business requirements. This approach is suitable for complex poorly-formalized systems under conditions of uncertainty, which is typical for the web service selection problem in SOA design for SME information systems. (Ma \& Zhang, 2010)

Well-known approaches to optimization of the web service composition in SOA (Zheng et al., 2011; Menasce, 2002; Ouzzani \& Bouguettaya, 2004; Hwang et al., 2007; Zheng et al., 2013; Maximilien \& Singh, 2004; Ardagna \& Pernici, 2007; Klein et al., 2014) don't take into account the integrated effect of various factors in conditions of uncertainty. In our approach the mapping of business processes to web services is specified by the fuzzy relation matrix whose elements are membership functions that determine how the web service meets the requirements of a specific business process. The restrictions are defined in accordance with permissible levels of service quality in the form of a $\Delta$-level subset of a fuzzy set.

We offer two types of web services selection criteria: for the best and the minimum sufficient conditions to ensure the information system quality. The proposed models allow the end-user to evaluate and select from a set of available services the service with the required functionality and quality more effectively in comparison with previous approaches. Some limitations of the effective application of the proposed approach should be pointed out. Firstly, it concerns business systems with limited set of business processes or limited set of available web services. Also more research efforts are needed to investigate the cases when business process implementation requires composite service that can specify many different business logics of its sub-services. The proposed models of web service selection in serviceoriented architecture when implemented as software, can be integrated in decision-making support system for design of SME information systems. The practical implementation of fuzzy models of web service selection in SOA system allows to generate data for further adaptation of the model by tuning the type and parameters of membership functions.

\section{Conclusion}

The goal of this study was to define principles for developing SOA architecture for small and middle enterprises (SME) using a fuzzy logic approach. The problem of assessing the quality of web services for optimal selection and system integration by taking into account the business requirements was presented. We have shown that web services quality assessment for their subsequent integration in service oriented information system should be based on the 


\section{Fuzzy Models of Web Services Selection in the Development of Service-Oriented Information Systems for Small and Medium Enterprises Authors: Irina Shpolianskaya et al.}

integrated quality index. This integrated quality index includes a set of indicators related to non-functional and functional requirements as well as web services user satisfaction indicators for evaluation of web service performance. In our research we have studied the use of the fuzzy logic methods to obtain an integrated assessment of the web services quality for their appropriate selection in SOA system in the conditions of incomplete information. The proposed fuzzy model and algorithms of web services selection provide the effective decision-support mechanisms for construction of SOA-based systems for small and middle enterprises.

\section{References}

Adelson, R.M. \& Fishburn, P.C. (1971). Utility theory for decision making. Operational Research Quarterly (1970-1977), 22(3), 308.

Ardagna, D. \& Pernici, B. (2007). Adaptive service composition in flexible processes. IEEE Transactions on Software Engineering, 33(6), 369-384.

Ardagna, D. \& Mirandola, R. (2010). Per-flow optimal service selection for Web services based processes. Journal of Systems and Software, 83(8), 1512-1523.

Ardagna, D. et al. (2011). A service-based framework for flexible business processes. IEEE Software, 28(2), 6167.

Dolzhenko, A. (2007). Information services model on the basis of fuzzy set theory. University news. NorthCaucasian region. Technical Sciences Series, 1, 7-10.

Hao, Y., Zhang, Y. \& Cao, J. (2010). Web services discovery and rank: An information retrieval approach. Future Generation Computer Systems, 26(8), 1053-1062.

Hwang, S. et al. (2007). A probabilistic approach to modeling and estimating the QoS of web-services-based workflows. Information Sciences, 177(23), 5484-5503.

Klein, A., Ishikawa, F. \& Honiden, S. (2014). SanGA: A self-adaptive network-aware approach to service composition. IEEE Transactions on Services Computing, 7(3), 452-464.

Kritikos, K. \& Plexousakis, D. (2009). Requirements for QoS-based web service description and discovery. IEEE Transactions on Services Computing, 2(4), 320-337.

Ma, Y. \& Zhang, C. (2008). Quick convergence of genetic algorithm for QoS-driven web service selection. Computer Networks, 52(5), 1093-1104.

Maximilien, E. \& Singh, M. (2004). A framework and ontology for dynamic web services selection. IEEE Internet Computing, 8(5), 84-93.

Menasce, D.A., Casalicchio, E. \& Dubey, V. (2010). On optimal service selection in Service Oriented Architectures. Performance Evaluation, 67(8), 659-675.

Menasce, D. (2002). QoS issues in web services. IEEE Internet Computing, 6(6), 72-75.

Ouzzani, M. \& Bouguettaya, A. (2004). Efficient access to web services. IEEE Internet Computing, 8(2), 34-44.

Papazoglou, M.P. \& Georgakopoulos, D. (2003). Introduction. Communications of the ACM, 46(10), 24.

Ray, A.W. \& Ray, J.J. (2006). Strategic benefits to SMEs from third party web services: An action research analysis. The Journal of Strategic Information Systems, 15(4), 273-291.

Rosario, S. et al. (2008). Probabilistic QoS and soft contracts for transaction-based web services orchestrations. IEEE Transactions on Services Computing, 1(4), 187-200.

Seta, P.A. \& Arman, A.A. (2014). General service oriented architecture (SOA) for small medium enterprise (SME). International Conference on ICT for Smart Society (ICISS), 217-221.

Skoutas, D. et al. (2010). Ranking and clustering web services using multicriteria dominance relationships. IEEE Transactions on Services Computing, 3(3), 163-177.

Stal, M. (2002). Web services: Beyond component-based computing. Communications of the ACM, 45(10), 7176.

Vidgen, R. et al. (2004). Web service business transformation: collaborative commerce opportunities in SMEs. Journal of Enterprise Information Management, 17(5), 372-381.

Wang, H., Lee, C. \& Ho, T. (2007). Combining subjective and objective QoS factors for personalized web service selection. Expert Systems with Applications, 32(2), 571-584.

Wang, P. (2009). QoS-aware web services selection with intuitionistic fuzzy set under consumer's vague perception. Expert Systems with Applications, 36(3), 4460-4466.

Wang, P., Chao, K. \& Lo, C. (2010). On optimal decision for QoS-aware composite service selection. Expert Systems with Applications, 37(1), 440-449. 
Yager, R.R. (1988). On ordered weighted averaging aggregation operators in multicriteria decision making. IEEE Transactions on Systems, Man and Cybernetics, 18, 183-190.

Yu, Q. \& Bouguettaya, A. (2010). Computing service skyline from uncertain QoWS. IEEE Transactions on Services Computing, 3(1), 16-29.

Yu, Q. \& Bouguettaya, A. (2011). Multi-attribute optimization in service selection. World Wide Web, 15(1), 131.

Yu, T., Zhang, Y. \& Lin, K. (2007). Efficient algorithms for web services selection with end-to-end QoS constraints. ACM Transactions on the Web, 1(1).

Zhang, W. et al. (2010). QoS-based dynamic web service composition with ant colony optimization. 2010 IEEE 34th Annual Computer Software and Applications Conference.

Zheng, Z. et al. (2011). QoS-aware web service recommendation by collaborative filtering. IEEE Transactions on Services Computing, 4(2), 140-152.

Zheng, H. et al. (2013). QoS analysis for web service compositions with complex structures. IEEE Transactions on Services Computing, 6(3), 373-386.

Zeng, L. et al. (2004). QoS-aware middleware for Web services composition. IEEE Transactions on Software Engineering, 30(5), 311-327. 